\title{
Dynamics of Micromechanical Coriolis Vibratory Gyroscopes
}

\author{
Vladislav Apostolyuk ${ }^{1}$ and Francis E. H. Tay ${ }^{2}$ \\ ${ }^{1}$ Micro and Nano Systems Cluster, Institute of Materials Research and Engineering, 117602 Singapore \\ ${ }^{2}$ Mechanical Engineering Department, National University of Singapore, 119602 Singapore
}

(Received: August 20, 2004. Accepted: August 23, 2004.)

\begin{abstract}
A general approach to the analysis of the dynamics of different types of micromechanical vibratory gyroscopes is considered in this paper. Specifically, we investigate and analyse the dynamics of singlemass gyroscopes for both fixed and rotating base. Generalized motion equations are derived and solved by means of method of averaging. Amplitude and phase responses of the sensitive element along with sensitivity to the angular rate are studied in detail. Micromechanical gyroscopes as an element of control systems are also considered in the paper.
\end{abstract}

Keywords: Vibratory, Gyroscope, Coriolis, Micromechanical, Dynamics.

\section{INTRODUCTION}

Fabrication technologies for microcomponents, microsensors, micromachines and micro-electromechanical systems (MEMS) are being rapidly developed, and represent a major research effort worldwide. There are many techniques currently being utilized in production of different types of MEMS including inertial micro-sensors and has made it possible to fabricate MEMS in high volumes at low individual cost.

Micro-mechanical Coriolis vibratory gyroscopes (CVG) have already been proposed for or actually deployed in numerous applications, including automotive active suspension and traction control systems, air bag activation, consumer electronics, guided munitions, robotics, etc. As the technology advances, micro-mechanical sensors will be deployed in many other functions that can benefit from the inexpensive detection of angular rate as well as other motion parameters. A key component of the angular rate sensor is a mechanical structure (or sensitive element) that is sensitive to rotation. One of the main problem sources in CVG development is quite intuitive design approach and almost absolute absence of the well-developed operation and error theories as well as analytical design methodologies. Currently designers have to make numerous simulations and experimental researches trying to find appropriate designs for sensitive elements. This approach only occasionally could result in an optimal and efficient design. As a result performances of all present micro-mechanical CVG still remain very low, which significantly reduces number of the possible applications.

One of the ways to improve performances of CVG is to analyse their dynamics and errors. Majority of the papers concerned with modelling of the micromechanical gyroscopes show results obtained either by finite element methods or by numerical solutions of the motion equations. However, such an approach only by chance leads to optimal designs of sensitive elements. Neither it allows development of error models and revealing various useful aspects of the sensitive element dynamics. Therefore, main emphasis of this paper is on development analytical, rather than numerical, model of the sensitive element.

Mathematical models of symmetrical (without decoupling frames) sensitive elements with translation movement of a proof mass applicable to analysis of CVG were considered in Ref. [1,2]. Dynamics and errors of CVG based on the sensitive element with decoupling frame have been studied in Ref. [3-5]. Further analysis of the dynamics and errors leads to analytical design methodologies of the sensitive element. ${ }^{6}$ 


\section{KINEMATIC SCHEMES AND OPERATION PRINCIPLES}

Most of all modern micro-mechanical gyroscopes utilize single proof mass that performs either translational or in some specific cases rotational oscillations. Comparing with double mass schemes one can see that single mass gyroscopes are advantageous from the fabrication simplicity and further miniaturizing points of view. Also translational displacements of the proof mass provide better measurement performances.

Currently there are two most widely used translational schemes of the single mass micro-mechanical gyroscopes: either with decoupling frame or without. Decoupling frame provides arbitrary choice of the sensitivity axis as well as reduces quadrature error caused by excitation system because of imperfect elastic suspension. It is essential for spatial angular rate measurement and for development of an inertial measurement unit.

Kinematic schemes of the single mass gyroscopes with decoupling frame and without are shown in Figure 1 and 2 correspondingly.

For the both shown kinematic schemes primary oscillations are excited along axis $X_{2}$. In case of rotation of the basis because of Coriolis inertia forces there will appear secondary oscillations along direction of the axis $X_{1}$. In the case of single mass gyroscope without decoupling frame spring have to provide well-tuned oscillations along two axes while for decoupling frame there is no such requirement for the same springs. This allows reducing fabrication tolerances requirements for the gyroscopes with decoupling frame.

From the mathematical modelling point of view scheme with decoupling frame is more general relative with respect to scheme without frame. Thus if assume mass of the decoupling frame equal to zero one can obtain kinematics without decoupling frame. Therefore during mathematical

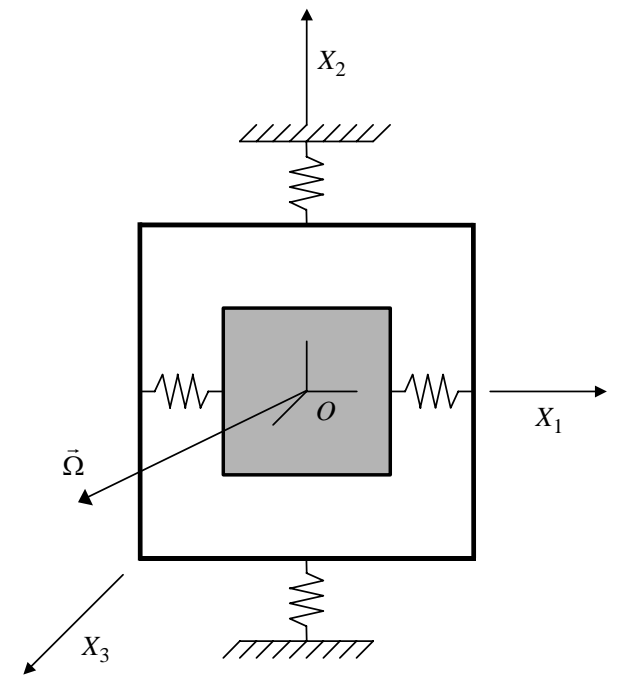

Figure 1. Kinematic scheme with decoupling frame.

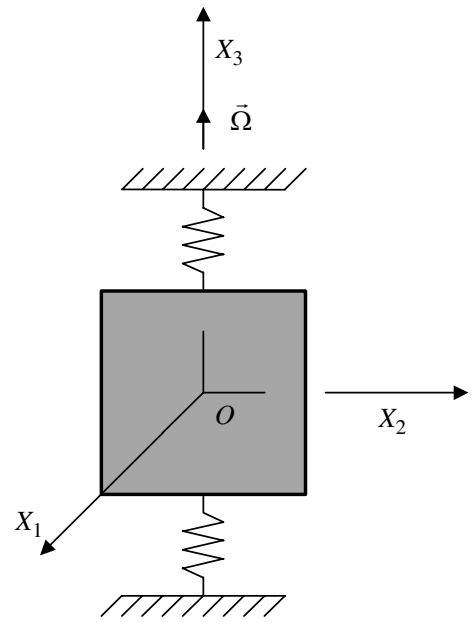

Figure 2. Kinematic scheme without decoupling frame.

analysis we can consider only scheme with decoupling frame as more general case. Theory of the single mass gyroscope without decoupling frame will be considered as a particular case of the general theory of the gyroscope with decoupling frame.

\section{MOTION EQUATIONS}

In order to obtain motion equation of the sensitive element of the single mass micro-mechanical gyroscope let us use Lagrange equations of the 2nd kind. Let us introduce orthogonal reference system $O X_{1} X_{2} X_{3}$ with origin at the gravity centre of the proof mass. Axis $X_{1}$ will be collinear with secondary (output) oscillations and axis $X_{2}$ will correspond to primary (excited) oscillations of the proof mass (see Fig. 1). Hence axis $X_{3}$ will be orthogonal to the plane of the sensitive element.

As a generalized coordinate $x_{2}$ (primary oscillations) let us assume displacements of the sensitive element along axis $X_{2}$. Similarly generalized coordinate $x_{1}$ will correspond to the displacements of the sensitive element along axis $X_{1}$. Here and after subscribe index mark number will refer to the number of the corresponding axis.

Basis, where sensitive element is installed, is assumed to rotate with arbitrary angular rate $\vec{\Omega}$ that is defined by its projections on the defined above reference frame as $\vec{\Omega}=$ $\left\{\Omega_{1}, \Omega_{2}, \Omega_{3}\right\}$. Position of the gravity centre of the proof mass is given by vector $\vec{r}_{1}=\left\{x_{1}, x_{2}, 0\right\}$. Position of the gravity centre of the decoupling frame is given by vector $\vec{r}_{2}=\left\{0, x_{2}, 0\right\}$. Hence absolute velocities of the mass and decoupling frame are given by following expressions

$$
\begin{aligned}
& \vec{V}_{1}=\left\{\dot{x}_{1}-x_{2} \Omega_{3}, \dot{x}_{2}+x_{1} \Omega_{3}, x_{2} \Omega_{1}-x_{1} \Omega_{2}\right\} \\
& \vec{V}_{2}=\left\{-x_{2} \Omega_{3}, \dot{x}_{2}, x_{2} \Omega_{1}\right\}
\end{aligned}
$$

where $\vec{V}_{1}$ is the absolute velocity of the proof mass, $\vec{V}_{2}$ is the absolute velocity of the decoupling frame. Taking 
into consideration (1) total kinetic energy of the sensitive element will be

$$
\begin{aligned}
T= & \frac{m_{1}}{2}\left[\left(\dot{x}_{1}-x_{2} \Omega_{3}\right)^{2}+\left(\dot{x}_{2}+x_{1} \Omega_{3}\right)^{2}+\left(x_{2} \Omega_{1}-x_{1} \Omega_{2}\right)^{2}\right] \\
& +\frac{m_{2}}{2}\left[x_{2}^{2} \Omega_{3}^{2}+\dot{x}_{2}^{2}+x_{2}^{2} \Omega_{1}^{2}\right]
\end{aligned}
$$

Here $m_{1}$ is the mass of the proof mass and $m_{2}$ is the mass of the decoupling frame. Potential energy of the sensitive element is formed by stiffness of its springs and is given by formula

$$
P=\frac{c_{1}}{2} x_{1}^{2}+\frac{c_{2}}{2} x_{2}^{2}
$$

where $c_{1}$ is the total stiffness of the elastic suspension along axis $X_{1}$ and $c_{2}$ is the total stiffness along axis $X_{2}$.

For the mechanical system with two degrees of freedom Lagrange's equations of the 2 nd kind will be

$$
\frac{d}{d t}\left(\frac{\partial L}{\partial \dot{x}_{i}}\right)-\frac{\partial L}{\partial x_{i}}=Q_{i} \quad(i=1,2)
$$

Here $L=T-P$ is the Lagrange's function, $Q_{i}$ are generalized forces caused by damping, actuation and so on.

Considering kinetic energy (2) and potential energy (3) and substituting them into equations (4) after simplifications one can obtain system of two differential equations that describe motion of the sensitive element of the single mass micro-mechanical gyroscope with decoupling frame

$$
\left\{\begin{array}{l}
\ddot{x}_{1}+\left(k_{1}^{2}-\Omega_{2}^{2}-\Omega_{3}^{2}\right) x_{1}-2 \Omega_{3} \dot{x}_{2}+\left(\Omega_{1} \Omega_{2}-\dot{\Omega}_{3}\right) x_{2}=q_{1} \\
\ddot{x}_{2}+\left(k_{2}^{2}-\Omega_{1}^{2}-\Omega_{3}^{2}\right) x_{2}+2 d \Omega_{3} \dot{x}_{1}+d\left(\Omega_{1} \Omega_{2}+\dot{\Omega}_{3}\right) x_{1}=q_{2}
\end{array}\right.
$$

where $k_{1}^{2}=c_{1} / m_{1}$ and $k_{2}^{2}=c_{2} /\left(m_{1}+m_{2}\right)$ are natural frequencies of secondary and primary oscillations, $d=$ $m_{1} /\left(m_{1}+m_{2}\right)$ is dimensionless inertia asymmetry factor, $q_{1}=Q_{1} / m_{1}, q_{2}=Q_{2} /\left(m_{1}+m_{2}\right)$ are generalized accelerations from external forces.

Note that if assume $d=1$ one can obtain motion equations for the single mass micro-mechanical gyroscope without decoupling frame.

Equation system (5) can be improved by introducing damping forces terms

$$
\left\{\begin{array}{l}
\ddot{x}_{1}+2 h_{1} \dot{x}_{1}+\left(k_{1}^{2}-\Omega_{2}^{2}-\Omega_{3}^{2}\right) x_{1}-2 \Omega_{3} \dot{x}_{2}+\left(\Omega_{1} \Omega_{2}-\dot{\Omega}_{3}\right) x_{2}=q_{1} \\
\ddot{x}_{2}+2 h_{2} \dot{x}_{2}+\left(k_{2}^{2}-\Omega_{1}^{2}-\Omega_{3}^{2}\right) x_{2}+2 d \Omega_{3} \dot{x}_{1}+d\left(\Omega_{1} \Omega_{2}+\dot{\Omega}_{3}\right) x_{1}=q_{2}
\end{array}\right.
$$

where $h_{1}$ and $h_{2}$ are damping factors that are correspond to the secondary and primary motion of the sensitive element.

It is apparent from analysis of the equations (6) that in case of ideal elastic suspension primary and secondary movement equations in this system are cross-coupled only by means of angular rate terms. It means that with absence any of the external forces acting on the proof mass along generalized coordinate $x_{1}$ any displacements in this direction will be caused by the angular rate. Detailed consideration of the equation system (6) reveals that the angular rate is not an input force but unknown parameter of the system.
In general case it makes system (6) consisting of linear equations but with variable coefficients. Usually it is quite complicated task to find closed form analytical solution of such system in the general case.

In order to simplify solution for some particular problems one can assume components of the angular rate vector $\vec{\Omega}$ in the reference system $O X_{1} X_{2} X_{3}$ as constants or quasiconstants. In this case equation system (6) can be modified and result system of linear differential equations with constant coefficients will be

$$
\left\{\begin{array}{l}
\ddot{x}_{1}+2 h_{1} \dot{x}_{1}+\left(k_{1}^{2}-\Omega_{2}^{2}-\Omega_{3}^{2}\right) x_{1}-2 \Omega_{3} \dot{x}_{2}+\Omega_{1} \Omega_{2} x_{2}=q_{1} \\
\ddot{x}_{2}+2 h_{2} \dot{x}_{2}+\left(k_{2}^{2}-\Omega_{1}^{2}-\Omega_{3}^{2}\right) x_{2}+2 d \Omega_{3} \dot{x}_{1}+d \Omega_{1} \Omega_{2} x_{1}=q_{2}
\end{array}\right.
$$

Solution of the system (7) describes behaviour of the sensitive element only for the constant angular rate. It has to be noted that all components of the angular rate $\vec{\Omega}$ except $\Omega_{3}$ are included only in non-linear way. It makes impossible to measure linearly other components of the angular rate except $\Omega_{3}$, which is orthogonal to the plane of primary and secondary oscillations. Any displacements of the sensitive element caused by other components of the angular rate have to be considered as cross-sensitivity errors. Let us assume zero all components of the angular rate vector that are not orthogonal to the plane of primary and secondary oscillations. In this case the angular rate vector will be $\vec{\Omega}=\{0,0, \Omega\}$ and system of equations (7) also will be simplified

$$
\left\{\begin{array}{l}
\ddot{x}_{1}+2 h_{1} \dot{x}_{1}+\left(k_{1}^{2}-\Omega^{2}\right) x_{1}-2 \Omega \dot{x}_{2}-\dot{\Omega} x_{2}=q_{1} \\
\ddot{x}_{2}+2 h_{2} \dot{x}_{2}+\left(k_{2}^{2}-\Omega^{2}\right) x_{2}+2 d \Omega \dot{x}_{1}+d \dot{\Omega} x_{1}=q_{2}
\end{array}\right.
$$

Further simplification could be done for the constant angular rate $\Omega=$ const

$$
\left\{\begin{array}{l}
\ddot{x}_{1}+2 h_{1} \dot{x}_{1}+\left(k_{1}^{2}-\Omega^{2}\right) x_{1}-2 \Omega \dot{x}_{2}=q_{1} \\
\ddot{x}_{2}+2 h_{2} \dot{x}_{2}+\left(k_{2}^{2}-\Omega^{2}\right) x_{2}+2 d \Omega \dot{x}_{1}=q_{2}
\end{array}\right.
$$

Considered obtained equation systems (8) and (9) one can see that for the small angular rates comparing with natural frequencies "non-linear" term $\Omega^{2}$ caused by centrifugical acceleration can be neglected that gives us possibility to perform further simplifications of the motion equations

$$
\left\{\begin{array}{l}
\ddot{x}_{1}+2 h_{1} \dot{x}_{1}+k_{1}^{2} x_{1}-2 \Omega \dot{x}_{2}=q_{1} \\
\ddot{x}_{2}+2 h_{2} \dot{x}_{2}+k_{2}^{2} x_{2}+2 d \Omega \dot{x}_{1}=q_{2}
\end{array}\right.
$$

Thus we derived basis set of motion equations of the sensitive element of the single mass micro-mechanical gyroscopes. These equations could be used for detailed analysis of any kind of gyroscopes with translational primary and secondary oscillations including both in-plane and out-plane designs. As was mentioned above in order to study dynamics of the sensitive element without decoupling frame the inertia asymmetry factor $d$ has to be assumed equal to unity. 


\section{MOTION OF THE SENSITIVE ELEMENT ON THE FIXED BASE}

Let us consider motion of the sensitive element of the micro-mechanical gyroscope on the fixed base. In this case the angular rate will be $\Omega=0$ and system of differential movement equations (10) will be simplified as following

$$
\left\{\begin{array}{l}
\ddot{x}_{1}+2 h_{1} \dot{x}_{1}+k_{1}^{2} x_{1}=0 \\
\ddot{x}_{2}+2 h_{2} \dot{x}_{2}+k_{2}^{2} x_{2}=q_{2}(t)
\end{array}\right.
$$

One can see that system of equations become set of two independent differential equations of the second order. In case of harmonic (sinusoidal) excitation with frequency $\omega$ and phase $\sigma$ generalized force acting on the sensitive element will be

$$
q_{2}(t)=q_{2} \sin (\omega t+\sigma)
$$

General closed form solution for the set of equations (12) is given by the following formulae

$$
\begin{aligned}
x_{1}(t)= & 0 \\
x_{2}(t)= & A_{2} \mathrm{e}^{-h_{2} t} \sin \left(t \sqrt{k_{2}^{2}-h_{2}^{2}}+\varphi_{2}\right) \\
& +\frac{q_{2}}{\sqrt{\left(k_{2}^{2}-\omega^{2}\right)^{2}+4 h_{2}^{2} \omega^{2}}} \sin (\omega t+\gamma)
\end{aligned}
$$

where constant coefficients $A_{2}$ and $\varphi_{2}$ can be determined from the initial conditions and partial solution phase $\gamma$ is determined as

$$
\operatorname{tg}(\sigma-\gamma)=2 h_{2} \omega /\left(k_{2}^{2}-\omega^{2}\right)
$$

Analysis of the obtained solution (13) and (14) shows us that frame of the sensitive element together with the proof mass performs forced oscillations with amplitude which is proportional to the excitation force (after natural oscillations will be damped) along axis $X_{2}$. In the same time proof mass remains unmovable along axis $X_{1}$ and output signal from the sensor will be zero. Furthermore subsiding time of the natural oscillations will determine start-up time of the micro-mechanical gyroscope, when sensitive element will oscillate with constant amplitude.

\section{MOTION OF THE SENSITIVE ELEMENT ON THE ROTATING BASE}

Let us study now behavior of the sensitive element of the micro-mechanical gyroscope on the base that rotates with constant angular rate. Without any generalization restrictions we can assume that vector of the constant angular rate is aligned with axis $X_{3}$ e.g. $\vec{\Omega}=\{0,0, \Omega\}$. In this case motion of the sensitive element will be determined as a solution of the system of differential equations (9) where $q_{1}(t)=0$. Unlike the set of equations (11) here we have system of equations that a cross-coupled by gyroscopic terms with the angular rate. With ideal elastic suspension of the proof mass secondary motion will depend only from the angular rate. Natural solution of the system (9) is obvious and is not interesting from the rate measurement point of view because all natural oscillation will damp in a short time. If excitation of the primary oscillation is harmonic the real excitation force in terms of the system (9) might be represented in complex form as $q_{2}(t)=\operatorname{Re}\left\{q_{2} \mathrm{e}^{i \omega t}\right\}$. Here $\omega$ is the excitation frequency and phase is assumed zero. Primary and secondary oscillations of the proof mass and the decoupling frame we shall determine as a particular solution of the system (9) in the following form

$$
\begin{array}{ll}
x_{1}(t)=\operatorname{Re}\left\{\bar{A}_{1} \mathrm{e}^{i \omega t}\right\} & \bar{A}_{1}=A_{1} \mathrm{e}^{i \varphi_{1}} \\
x_{2}(t)=\operatorname{Re}\left\{\bar{A}_{2} \mathrm{e}^{i \omega t}\right\} & \bar{A}_{2}=A_{2} \mathrm{e}^{i \varphi_{2}}
\end{array}
$$

where $A_{1}$ and $A_{2}$ are the amplitudes of the translational secondary and primary oscillations correspondingly, $\varphi_{1}$ and $\varphi_{2}$ are their phases. After substitution of the expressions (15) to the system (9) another system but linear algebraic equations of unknown complex variables $\bar{A}_{1}$ and $\bar{A}_{2}$ is obtained

$$
\left\{\begin{array}{l}
\left(k_{1}^{2}-\Omega^{2}-\omega^{2}+2 h_{1} i \omega\right) \bar{A}_{1}-2 \Omega i \omega \bar{A}_{2}=0 \\
2 d \Omega i \omega \bar{A}_{1}+\left(k_{2}^{2}-\Omega^{2}-\omega^{2}+2 h_{2} i \omega\right) \bar{A}_{2}=q_{2}
\end{array}\right.
$$

Principal determinant of this system is given as

$$
\begin{aligned}
\bar{\Delta}\left(\omega, \Omega^{2}\right)= & \left(k_{1}^{2}-\Omega^{2}-\omega^{2}\right)\left(k_{2}^{2}-\Omega^{2}-\omega^{2}\right) \\
& -4 \omega^{2}\left(h_{1} h_{2}+d \Omega^{2}\right) \\
& +2 i \omega\left[h_{1}\left(k_{2}^{2}-\Omega^{2}-\omega^{2}\right)+h_{2}\left(k_{1}^{2}-\Omega^{2}-\omega^{2}\right)\right]
\end{aligned}
$$

Solution of the system (16) determined by Kramer's method is

$$
\bar{A}_{1}=\frac{2 q_{2} i \omega}{\bar{\Delta}\left(\omega, \Omega^{2}\right)} \Omega \quad \bar{A}_{2}=\frac{q_{2}\left(k_{1}^{2}-\Omega^{2}-\omega^{2}+2 h_{1} i \omega\right)}{\bar{\Delta}\left(\omega, \Omega^{2}\right)}
$$

Using of the complex amplitude method (modification of the averaging method) is allowing us not to analyze displacements of the sensitive element but to analyze directly amplitudes and phases that are of great interest from the vibratory gyroscope point of view. From the analysis of the (17) one can see that excitation of the primary oscillations will provide the amplitude of the secondary oscillations be almost linear function of the unknown angular rate. Therefore in order to determine parameters of the oscillations let us determine their amplitudes and phases. Conversion from the complex amplitudes to the real amplitudes and phases is performed as follows

$$
A=|\bar{A}|=\sqrt{\operatorname{Re}(\bar{A})^{2}+\operatorname{Im}(\bar{A})^{2}} \quad \operatorname{tg}(\varphi)=\frac{\operatorname{Im}(\bar{A})}{\operatorname{Re}(\bar{A})}
$$


This transformation gives us following formulae for the primary and secondary amplitudes

$$
\begin{aligned}
A_{1}= & \frac{2 q_{2} \omega}{\Delta} \Omega \quad A_{2}=\frac{q_{2} \sqrt{\left(k_{1}^{2}-\Omega^{2}-\omega^{2}\right)^{2}+4 h_{1}^{2} \omega^{2}}}{\Delta} \\
\Delta^{2}= & {\left[\left(k_{1}^{2}-\Omega^{2}-\omega^{2}\right)\left(k_{2}^{2}-\Omega^{2}-\omega^{2}\right)\right.} \\
& \left.-4 \omega^{2}\left(h_{1} h_{2}+d \Omega^{2}\right)\right]^{2} \\
& +4 \omega^{2}\left[h_{1}\left(k_{2}^{2}-\Omega^{2}-\omega^{2}\right)+h_{2}\left(k_{1}^{2}-\Omega^{2}-\omega^{2}\right)\right]^{2}
\end{aligned}
$$

and also their phases

$$
\begin{gathered}
\operatorname{tg}\left(\varphi_{1}\right)=\frac{\left(k_{1}^{2}-\Omega^{2}-\omega^{2}\right)\left(k_{2}^{2}-\Omega^{2}-\omega^{2}\right)-4\left(h_{1} h_{2}+d \Omega^{2}\right) \omega^{2}}{2 \omega\left[h_{1}\left(k_{2}^{2}-\Omega^{2}-\omega^{2}\right)+h_{2}\left(k_{1}^{2}-\Omega^{2}-\omega^{2}\right)\right]} \\
\operatorname{tg}\left(\varphi_{2}\right)=\frac{b_{1}+b_{2}}{b_{3}-b_{4}} \\
b_{1}=2 \omega\left(k_{1}^{2}-\Omega^{2}-\omega^{2}\right)\left[h_{1}\left(k_{2}^{2}-\Omega^{2}-\omega^{2}\right)\right. \\
\left.\quad+h_{2}\left(k_{1}^{2}-\Omega^{2}-\omega^{2}\right)\right] \\
b_{2}=2 \omega h_{1}\left[\left(k_{1}^{2}-\Omega^{2}-\omega^{2}\right)\left(k_{2}^{2}-\Omega^{2}-\omega^{2}\right)\right. \\
\left.\quad-4 \omega^{2}\left(h_{1} h_{2}+d \Omega^{2}\right)\right] \\
b_{3}=\left(k_{1}^{2}-\Omega^{2}-\omega^{2}\right)\left[\left(k_{1}^{2}-\Omega^{2}-\omega^{2}\right)\left(k_{2}^{2}-\Omega^{2}-\omega^{2}\right)\right. \\
\left.\quad-4 \omega^{2}\left(h_{1} h_{2}+d \Omega^{2}\right)\right] \\
b_{4}=4 h_{1} \omega^{2}\left[h_{1}\left(k_{2}^{2}-\Omega^{2}-\omega^{2}\right)+h_{2}\left(k_{1}^{2}-\Omega^{2}-\omega^{2}\right)\right]
\end{gathered}
$$

Obtained expressions (20) are allowing us to analyse how amplitudes and phases of the primary and secondary oscillations depend on the angular rate. Graphic plot of the secondary amplitude as a function of the angular rate is shown in Figure 3. Excitation frequency was assumed to be equal to the natural frequency of the primary oscillations.

From the Figure 3 one can see that linear measurement of the angular rate is possible only in a range that is significantly smaller then natural frequencies of the sensitive element. In practice we can always choose and design our sensitive element properly in order to provide necessary linear measurement range for the micro-gyroscope.

\section{STABILITY OF THE OSCILLATIONS OF THE SENSITIVE ELEMENT}

One of the most essential aspect of any oscillatory system is its stability. Let us study stability of the oscillations of the

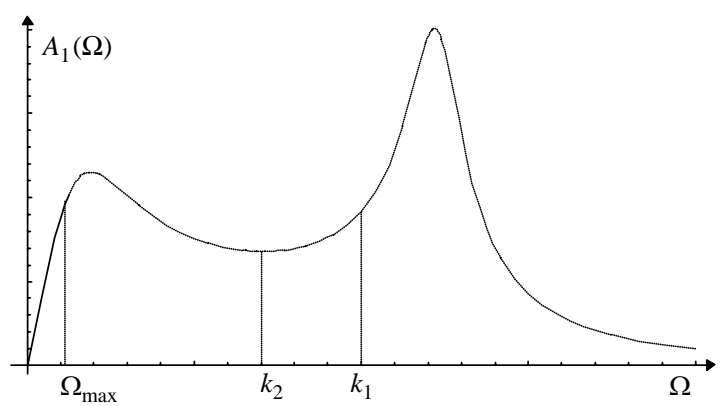

Figure 3. Secondary amplitude as a function of the angular rate. sensitive element of the micro-mechanical gyroscope with the decoupling frame that are caused by the angular rate. After Laplace transformation with zero initial conditions system (9) will be

$$
\left\{\begin{array}{l}
\left(p^{2}+2 h_{1} p+k_{1}^{2}-\Omega^{2}\right) X_{1}(p)-2 \Omega p X_{2}(p)=0 \\
2 d \Omega p X_{1}(p)+\left(p^{2}+2 h_{2} p+k_{2}^{2}-\Omega^{2}\right) X_{2}(p)=Q_{2}(p)
\end{array}\right.
$$

Characteristic equation of this system (21) is as following

$$
\begin{aligned}
& \Delta(p)=p^{4}+a_{3} p^{3}+a_{2} p^{2}+a_{1} p+a_{0}=0 \\
a_{3}= & 2\left(h_{1}+h_{2}\right) \quad a_{2}=4 h_{1} h_{2}+k_{1}^{2}+k_{2}^{2}+2(2 d-1) \Omega^{2} \\
a_{1}= & 2\left[h_{1}\left(k_{2}^{2}-\Omega^{2}\right)+h_{2}\left(k_{1}^{2}-\Omega^{2}\right)\right] \\
a_{0}= & k_{1}^{2} k_{2}^{2}-\left(k_{1}^{2}+k_{2}^{2}\right) \Omega^{2}+\Omega^{4}
\end{aligned}
$$

In order to analyse stability of this system we used Routh criteria that for our system is given by

$$
a_{1} a_{2} a_{3}-a_{1}^{2}-a_{3}^{2} a_{0}>0, \quad a_{i}>0, \quad i=0 \ldots 3
$$

Coefficients of the characteristic equation (22) are functions of the angular rate, damping factors and natural frequencies of the system. Only angular rate is unknown. All other parameters are subjects to design in accordance to the stability conditions (23). From analysis of (22) follows that only coefficients $a_{1}$ and $a_{0}$ could become negative. Besides lost of stability is possible also in the first expression in (23). Extreme stability conditions are given by following equations

$$
\begin{gathered}
a_{0}=k_{1}^{2} k_{2}^{2}-\left(k_{1}^{2}+k_{2}^{2}\right) \Omega^{2}+\Omega^{4}=0 \\
a_{1}=2\left[h_{1}\left(k_{2}^{2}-\Omega^{2}\right)+h_{2}\left(k_{1}^{2}-\Omega^{2}\right)\right]=0 \\
a_{1} a_{2} a_{3}-a_{1}^{2}-a_{3}^{2} a_{0}=0
\end{gathered}
$$

Solutions of the first equations from (24) are

$$
\Omega_{1,2}^{(0)}= \pm k_{1} \quad \Omega_{3,4}^{(0)}= \pm k_{2}
$$

Solutions of the second equation from (24) are given by

$$
\Omega_{1,2}^{(1)}= \pm \sqrt{\frac{h_{2} k_{1}^{2}+h_{1} k_{2}^{2}}{h_{1}+h_{2}}}
$$

Range where coefficients $a_{0}$ and $a_{1}$ are positive can be easily determined from graphic plots in Figures 4 and 5 .

Expressions for solution of the third equation from (24) are quite complicated and can be neglected as soon as their solutions are significantly larger than previous solutions. From the analysis of the formulae (25), (26) and Figures 4 and 5 following stability conditions can be derived

$$
-k_{2}<\Omega<k_{2}
$$

Thus stabile secondary oscillations of the sensitive element exist only for the angular which absolute value is less than the natural frequency of the primary oscillations. Relationships (27) have to be taken into consideration during designing of the sensitive element and be based on the required measurement range. 


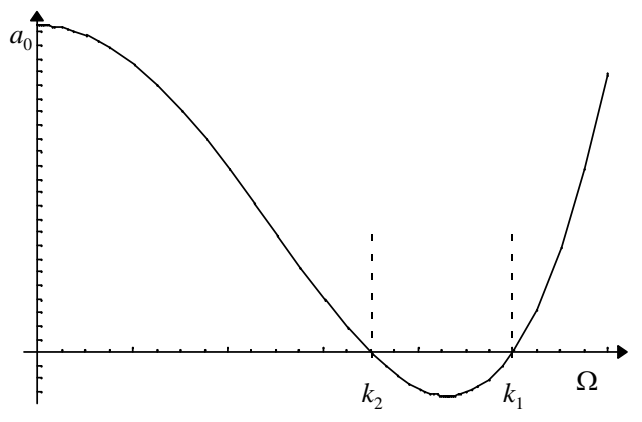

Figure 4. Coefficient $a_{0}$ as a function of the angular rate.

\section{EIGENFREQUENCIES OF THE SENSITIVE ELEMENT}

Eigenfrequencies of the oscillations of the sensitive element can be determined as roots of the characteristic equation (22). Roots of the characteristic equation can be determined approximately if damping can be neglected e.g. $a_{1}=$ $a_{3}=0$. In this case characteristic equation will be simplified to biquadratic

$$
\begin{gathered}
p^{4}+b_{1} p^{2}+b_{0}=0 \\
b_{1}=k_{1}^{2}+k_{2}^{2}+2(2 d-1) \Omega^{2} \\
b_{0}=\left(k_{1}^{2}-\Omega^{2}\right)\left(k_{2}^{2}-\Omega^{2}\right)=k_{1}^{2} k_{2}^{2}-\left(k_{1}^{2}+k_{2}^{2}\right) \Omega^{2}+\Omega^{4}
\end{gathered}
$$

Roots of the equation (28) are relating to the eigenfrequencies $\omega_{j 0}$ of the primary and secondary oscillations as

$$
p_{1,2}= \pm i \omega_{10} \quad p_{3,4}= \pm i \omega_{20}
$$

Hence solution of the equation (28) taking into consideration (29) will be

$$
\begin{aligned}
& \omega_{j 0}^{2}= \frac{1}{2}\left[k_{1}^{2}+k_{2}^{2}+2(2 d-1) \Omega^{2}\right] \\
&-\frac{(-1)^{j}}{2} \sqrt{\left[k_{1}^{2}+k_{2}^{2}+2(2 d-1) \Omega^{2}\right]^{2}-4\left(k_{1}^{2}-\Omega^{2}\right)\left(k_{2}^{2}-\Omega^{2}\right)} \\
& j=1,2 ; \quad \omega_{10}>\omega_{20}
\end{aligned}
$$

Principal graphical plot of the eigenfrequencies as a function of the angular rate is shown in Figure 6. In Figure 7 are shown derivations of the eigenfrequencies.

Let us now find eigenfrequencies of oscillations of the sensitive element without neglecting of the damping but

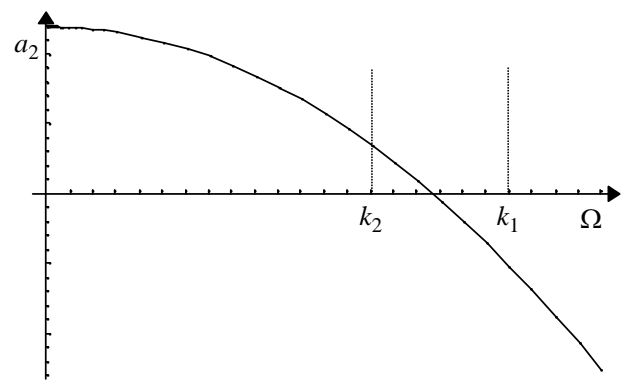

Figure 5. Coefficient $a_{1}$ as a function of the angular rate.

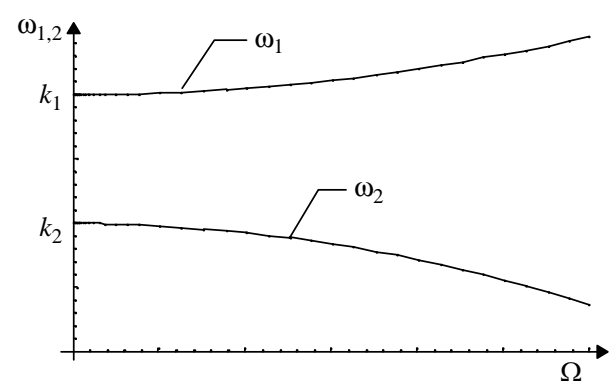

Figure 6. Eigenfrequencies as function of the angular rate.

assuming that damping is relatively small. Characteristic equation (22) can be represented as

$$
\Delta(p)=\left(p^{2}+2 h_{10}+\omega_{1}^{2}\right)\left(p^{2}+2 h_{20}+\omega_{2}^{2}\right)
$$

Here $\omega_{j}$ are the eigenfrequencies with small damping, $h_{j 0}$ are the small damping factors. After expanding of the equation (31) and equalizing factors of the same power of the $p$ we obtained system of four equations with unknown variables $\omega_{1}, \omega_{2}, h_{10}$ and $h_{20}$. Considering small damping factors roots of the characteristic equation will be represented be expressions

$$
p_{1,2}=-h_{10} \pm i \omega_{1} \quad p_{3,4}=-h_{20} \pm i \omega_{2}
$$

where $h_{10}=\left(a_{3} \omega_{10}^{2}-a_{1}\right) / 2\left(\omega_{10}^{2}-\omega_{20}^{2}\right) ; h_{20}=\left(a_{1}-a_{3} \omega_{20}^{2}\right) /$ $2\left(\omega_{10}^{2}-\omega_{20}^{2}\right) ; \omega_{1} \approx \omega_{10} ; \omega_{2} \approx \omega_{20}$.

One can note that formula (30) is relatively complicated. It can be simplified by expanding into the Taylor series. Preserving parabolic nature of the initial dependence gives us following formulae for the eigenfrequencies

$$
\begin{gathered}
\omega_{20}^{2} \approx \frac{b_{0}}{b_{1}}=\frac{\left(k_{1}^{2}-\Omega^{2}\right)\left(k_{2}^{2}-\Omega^{2}\right)}{k_{1}^{2}+k_{2}^{2}+2(2 d-1) \Omega^{2}} \\
\omega_{10}^{2} \approx b_{1}-\frac{b_{0}}{b_{1}}=k_{1}^{2}+k_{2}^{2}+2(2 d-1) \Omega^{2}-\omega_{20}^{2}
\end{gathered}
$$

Calculations of the eigenfrequencies based on these formulae are less computationally expensive comparing with (30) but gives only approximate results. Their calculation errors are in the range of $2 \%$ from the exact values.

There is another simpler and more precise method for calculation of the eigenfrequencies of the sensitive element

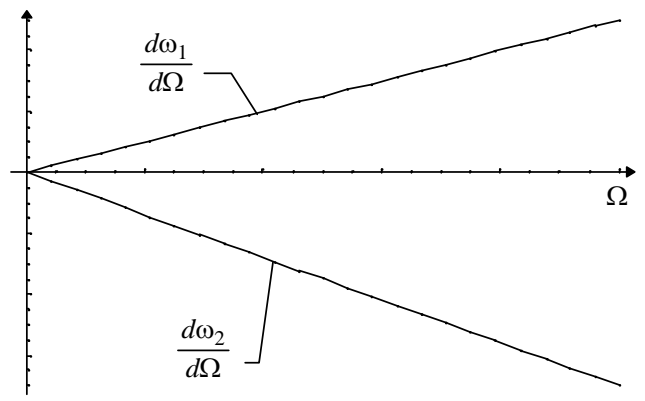

Figure 7. First derivatives from eigenfrequencies as functions of the angular rate. 
of the micro-mechanical gyroscopes. Since first derivatives of the egenfrequencies it is easy to see that they are almost linear functions of the angular rate (see Fig. 7). It appears from this that the eigenfrequencies dependent on the angular rate as a quadratic function. Hence we can search for solution of the characteristic equation in the following form

$$
\omega_{j}(\Omega)=k_{j}+l_{j} \frac{\Omega^{2}}{2}
$$

where $j=1,2 ; l_{j}$ are unknown constant coefficients that depend only on design parameters of the sensitive element. Determination of the constant coefficients $l_{j}$ is quite apparent because from the formula (33) follows $\left(\omega_{0 j}^{2}\right)_{\Omega=0}=k_{j}^{2}$. Another relationship can be obtained by differentiating of the equation (34), which gives us $\left(d \omega_{j} / d \Omega\right)=l_{j} \Omega$. Since $l_{j}$ don't depend on the angular rate we assume $\Omega=1$ and obtain that $l_{j}=\left(d \omega_{j} / d \omega\right)_{\Omega=1}$. After expanding and simplifying we obtained final formula for calculation of the constant coefficients $l_{j}$

$$
l_{j}=\left(\frac{s_{3}}{2}+\frac{(-1)^{j}}{2} \frac{2 s_{2}-s_{1} s_{3}}{\sqrt{s_{1}^{2}-4 s_{0}}}\right) / \sqrt{2 s_{1}-(-1)^{j} 2 \sqrt{s_{1}^{2}-4 s_{0}}}
$$

where $s_{0}=\left(k_{1}^{2}-1\right)\left(k_{2}^{2}-1\right), s_{1}=k_{1}^{2}+k_{2}^{2}+2(2 d-1), s_{2}=$ $2\left(2-k_{2}^{2}-k_{1}^{2}\right), s_{3}=4(2 d-1)$.

If natural frequencies are close enough to each other $\left(k_{1} \approx k_{2}\right)$ then the eigenfrequencies will depend on the angular rate in a linear way. In this case formula (34) will be transformed to

$$
\omega_{j}(\Omega)=k_{j}+l_{j} \Omega
$$

where $j=1,2$ and the constant coefficient $l_{j}$ determines from (35) where the natural frequencies have to be assumed equal $k_{1}=k_{2}$.

Resulting simple expressions for the eigenfrequencies allow us to calculate them precise and in the same time not computationally expensive. This can be used in a digital signal processing of the output information from the microgyroscope. Particularly for the algorithmic compensation of the errors caused by shifting of the eigenfrequencies.

\section{AMPLITUDE AND PHASE RESPONSES}

Motion of the sensitive element of the micro-mechanical gyroscope with decoupling frame on the basis that rotates with a constant angular rate $\vec{\Omega}=\{0,0, \Omega\}$ is described by the system of the differential Equations (9). After Laplace's transformation under zero initial conditions one can obtain

$$
\left\{\begin{array}{l}
\left(p^{2}+2 h_{1} p+k_{1}^{2}-\Omega^{2}\right) X_{1}(p)-2 \Omega p X_{2}(p)=0 \\
2 d \Omega p X_{1}(p)+\left(p^{2}+2 h_{2} p+k_{2}^{2}-\Omega^{2}\right) X_{2}(p)=Q_{2}(p)
\end{array}\right.
$$

By means of transfer functions system (37) can be represented schematically as shown in a block diagram in Figure 8.
Transfer functions in Figure 8 are given as

$$
\begin{gathered}
W_{1}(p)=\frac{1}{p^{2}+2 h_{1} p+k_{1}^{2}-\Omega^{2}} \\
W_{2}(p)=\frac{1}{p^{2}+2 h_{2} p+k_{2}^{2}-\Omega^{2}} \\
W_{g 1}(p)=2 p \Omega \quad W_{g 2}(p)=2 d p \Omega
\end{gathered}
$$

Excitation force is considered as input in the block diagram in the Figure 8. In this representation the angular rate is not an input of the system but is an unknown parameter. Nevertheless during selection of the working frequency it is necessary to maximize total gain on this parameter since in fact exactly this signal is modulated by measured value of the angular rate. Secondary oscillations are considered as output on the shown block diagram. Hence the principal block diagram can be simplified to the input-output form as

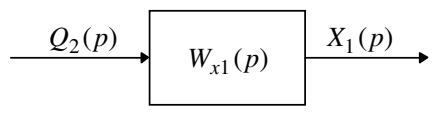

For this diagram

$$
\begin{aligned}
W_{x 1}(p) & =\frac{X_{1}(p)}{Q_{2}(p)} \\
& =\frac{2 p \Omega}{\left(p^{2}+2 h_{1} p+k_{1}^{2}-\Omega^{2}\right)\left(p^{2}+2 h_{2} p+k_{2}^{2}-\Omega^{2}\right)+4 p^{2} \Omega^{2} d}
\end{aligned}
$$

By means of variable substitution $p=i \omega$ we can derive a complex transfer function of our system that is given in a form

$W_{x 1}(i \omega)$

$$
=\frac{2 i \omega \Omega}{\left(k_{1}^{2}-\Omega^{2}-\omega^{2}+2 h_{1} i \omega\right)\left(k_{2}^{2}-\Omega^{2}-\omega^{2}+2 h_{2} i \omega\right)-4 \omega^{2} \Omega^{2} d}
$$

It has to be noted that resulted from (39) expression (40) is equivalent to the solution (17) for the complex amplitude $\bar{A}_{1}$. Formulae for the amplitude and phase responses of the secondary oscillations are following

$$
\begin{gathered}
A_{1}(\omega)=\frac{2 \omega}{\Delta} \Omega \\
\Delta^{2}=\left[\left(k_{1}^{2}-\Omega^{2}-\omega^{2}\right)\left(k_{2}^{2}-\Omega^{2}-\omega^{2}\right)-4 \omega^{2}\left(h_{1} h_{2}+d \Omega^{2}\right)\right]^{2} \\
+4 \omega^{2}\left[h_{1}\left(k_{2}^{2}-\Omega^{2}-\omega^{2}\right)+h_{2}\left(k_{1}^{2}-\Omega^{2}-\omega^{2}\right)\right]^{2} \\
\varphi_{1}(\omega)=\operatorname{arctg}\left(\frac{\left(k_{1}^{2}-\Omega^{2}-\omega^{2}\right)\left(k_{2}^{2}-\Omega^{2}-\omega^{2}\right)-4\left(h_{1} h_{2}+d \Omega^{2}\right) \omega^{2}}{2 \omega\left[h_{1}\left(k_{2}^{2}-\Omega^{2}-\omega^{2}\right)+h_{2}\left(k_{1}^{2}-\Omega^{2}-\omega^{2}\right)\right]}\right)
\end{gathered}
$$

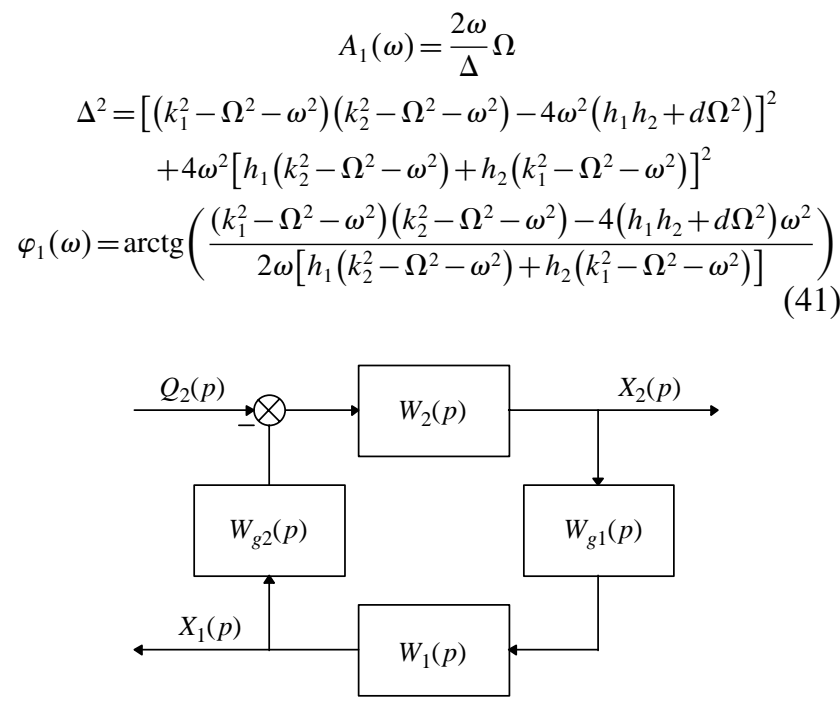

Figure 8. Basis block diagram of the sensitive element of the micro-

mechanical gyroscope. 


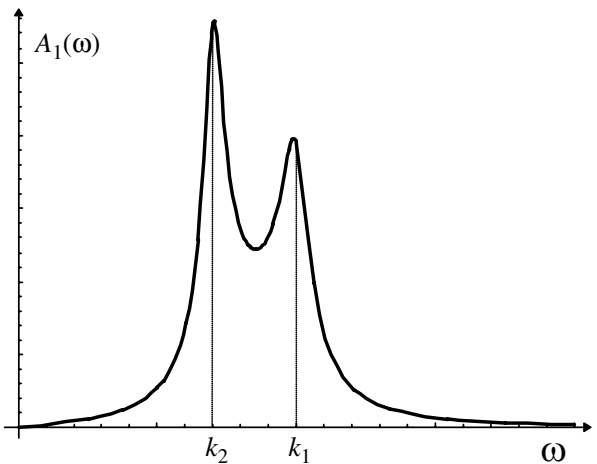

Figure 9. Amplitude response of the secondary oscillations.

Amplitude and phase responses of the secondary oscillations of the sensitive element are shown in Figures 9 and 10 correspondingly.

For the best sensitivity of the gyroscope to the angular rate it is reasonable to design its sensitive element in a way when its values of the eigenfrequencies $\omega_{1}$ and $\omega_{2}$ were close to each other as much as possible. As it can be seen from the graphic plot in the Figure 9 secondary oscillations of the sensitive element when excitation is performed in a resonance with the eigenfrequency of the primary oscillations. Therefore in order to increase sensitivity of the gyroscope it is necessary to perform excitation of the sensitive element at frequency that is close or equal to the lowest natural frequency of the system.

Let us consider now another output of the system presented in Figure 8 that corresponds to the primary oscillations of the sensitive element along axis $X_{2}$. For that we have to reduce our initial principal diagram to the following form

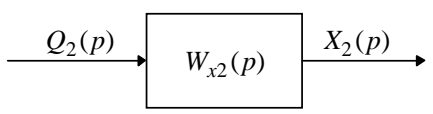

In this case transfer function $W_{x 2}(p)$ will be given by

$$
\begin{aligned}
W_{x 2}(p) & =\frac{X_{2}(p)}{Q_{2}(p)} \\
& =\frac{p^{2}+2 h_{1} p+k_{1}^{2}-\Omega^{2}}{\left(p^{2}+2 h_{1} p+k_{1}^{2}-\Omega^{2}\right)\left(p^{2}+2 h_{2} p+k_{2}^{2}-\Omega^{2}\right)+4 p^{2} d \Omega^{2}}
\end{aligned}
$$

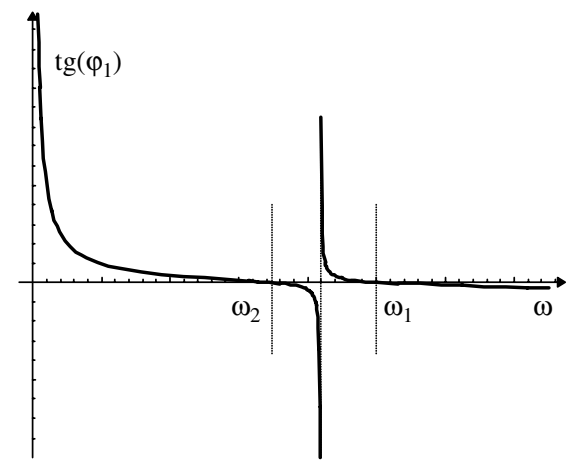

Figure 10. Phase response of the secondary oscillations.

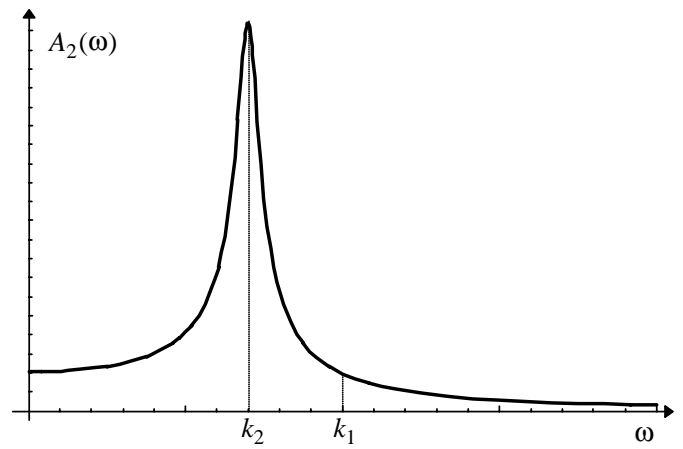

Figure 11. Amplitude response of the primary oscillations.

By substitution $p=i \omega$ the transfer function will be transformed to its complex representation

$$
\begin{aligned}
& W_{x 2}(i \omega) \\
& \quad=\frac{k_{1}^{2}-\Omega^{2}-\omega^{2}+2 h_{1} i \omega}{\left(k_{1}^{2}-\Omega^{2}-\omega^{2}+2 h_{1} i \omega\right)\left(k_{2}^{2}-\Omega^{2}-\omega^{2}+2 h_{2} i \omega\right)-4 \omega^{2} d \Omega^{2}}
\end{aligned}
$$

Formulae for the amplitude response of the primary oscillations is as follows

$$
A_{2}(\omega)=\sqrt{\frac{\left(k_{1}^{2}-\Omega^{2}-\omega^{2}\right)^{2}+4 h_{1}^{2} \omega^{2}}{\Delta^{2}}}
$$

Graphical plot of the amplitude of the primary oscillations is presented in Figure 11.

From analysis of the plot in Figure 11 one can see that influence of the secondary oscillations on the primary is negligible small and doesn't cause any detectable changes in the primary motion. This allows us to neglect by the Coriolis back cross coupling in some cases where it could be convenient and will not cause additional restrictions to the problem formulation.

\section{CONCLUSION}

The presented analysis of the sensitive element of micromechanical Coriolis vibratory gyroscopes allows both prediction of the performances and determination of the design parameters that are necessary to achieve high performance of inertial instruments. Even though the proposed approach is applied to sensitive elements, most of the dependencies can also be used for detailed analysis of the dynamics of CVG while designing control circuits.

\section{References and Notes}

1. B. Friedland and M. F. Hutton, IEEE Trans. on Automatic Control 4, 545 (1978).

2. D. Lynch, Proc. of 2nd St. Petersburg Conf. on Gyroscopic Technology and Navigation 26 (1995).

3. V. Apostolyuk and A. Zbrutsky, Proc. of the 4th St. Petersburg International Conf. on Integrated Navigation Systems 330 (1997).

4. V. Apostolyuk and A. Zbrutsky, Gyroscopy and Navigation 3, 13 (1998).

5. V. Apostolyuk and A. Zbrutsky, Scientific News of the National Technical University of Ukraine 1, 114 (1999).

6. V. Apostolyuk, V. J. Logeeswaran, and F. E. H. Tay, Journal of Micromechanics and Microengineering 12, 948 (2002). 